\title{
Thermoelasticity in Ge due to nonuniform distribution of doping impurity studied by light polarization modulation technique
}

\author{
B. K. Serdega, Ye. F. Venger, Ye. V. Nikitenko \\ Institute of Semiconductor Physics, National Academy of Sciences of Ukraine, Kyiv, 252028, Ukraine, \\ phone (044) 265 4020, e-mail: bshl@polarget.semicond.kiev.ua
}

\begin{abstract}
Volume-gradient photovoltage and birefrigence caused by a difference of main mechanical stress components have been studied in the Ge-monocrystal with step-like distribution of the doping impurity concentration, $N$. The qualitative agreement between the function obtained by integration of the spatial dependence of the anisotropy and of the coordinate dependence of the photovoltage was observed. Using this fact, the conclusion is made that the features of thermoelastic mechanical stress can be distribution of expressed by the dependence $\left|\sigma_{z}-\sigma_{y}\right| \sim d^{2} N / d z^{2}$.
\end{abstract}

Keywords: double refraction, polarization, modulation, photovoltage, anisotropy, thermal stress. Paper received 27.01.99; revised manuscript received 08.04.99; accepted for publication 19.04.99.

1. It is well-known that defects in the crystal lattice lead to the appearance of local deformations. Also it is known that the group of defects originated from doping and background impurities, or some technological causes, distributed nonuniformly in an ordered way, can result in the appearance of macroscopic deformations [1] It means that the ordered nonuniformities which, in real crystals, are created by the directed or uncontrolled doping, create a nonzero difference of main stress components at distances much longer than the lattice parameter. At the same time, a quantitative or, at least, a functional connection between parameters of the spatial distribution of defect concentration and the magnitudes of deformation-induced mechanical stresses remains to be studied yet. To the best of our knowledge, the works ascertaining the relation between distribution of residual (intrinsic) mechanical stresses in a crystal and the characteristics of nonuniformities of composition are absent. The only exception is the work [2] where the distribution of stress is studied for the particular case of the doping impurity diffusion from the crystal surface in the z-axis direction.

It can be supposed, extending the model of thermal stresses caused by a temperature gradient [3], that the mechanical stress, $\sigma$, occurring in the nonuniform concentration field (an analog of a temperature field) is proportional to the magnitude $d^{2} N / d x^{2}$, where $N$ is the distribution function of the defect (a doping impurity) concentration. Such an analogy can be based on a fact that if the mean interatomic distance depends on the doping impurity concentration (composition of the compound), then its spatial variation is accompanied by the appear- 


\section{B. K. Serdega et al.: Thermoelasticity in Ge due to nonuniform distribution ...}

ance of the uncompensated deformation component. If this is the case, then, taking into account the macroscopic scale of nonuniformities, it appears to be possible to study their spatial distribution using one of known techniques. This is the aim of the present study.

Its idea consists in the following. In a sample of a semiconductor crystal with a step-like coordinate dependence of the electrically active doping impurity the respective distribution of the volume-gradient photovoltage is measured [4] and compared with the experimentally found coordinate dependence of the value of the main component (Fig. 1). If the assumption is valid that the dependence of the stress in the nonunformity region has the form $\sigma \sim d^{2} N / d z^{2}$, then the function obtained by the double integration of the function $\sigma(z)$ (Fig. 1c), and the integral of the volume-gradient photovoltage $E(z)$ (Fig. 1b) should coincide with the function $N(z)$ (Fig. 1a).

2. Presently, various experimental methods are used for measurements of stressed-deflected mode of anisotropic solids: tensometry using the test structures, holographic interferometry, optopolarization method in a
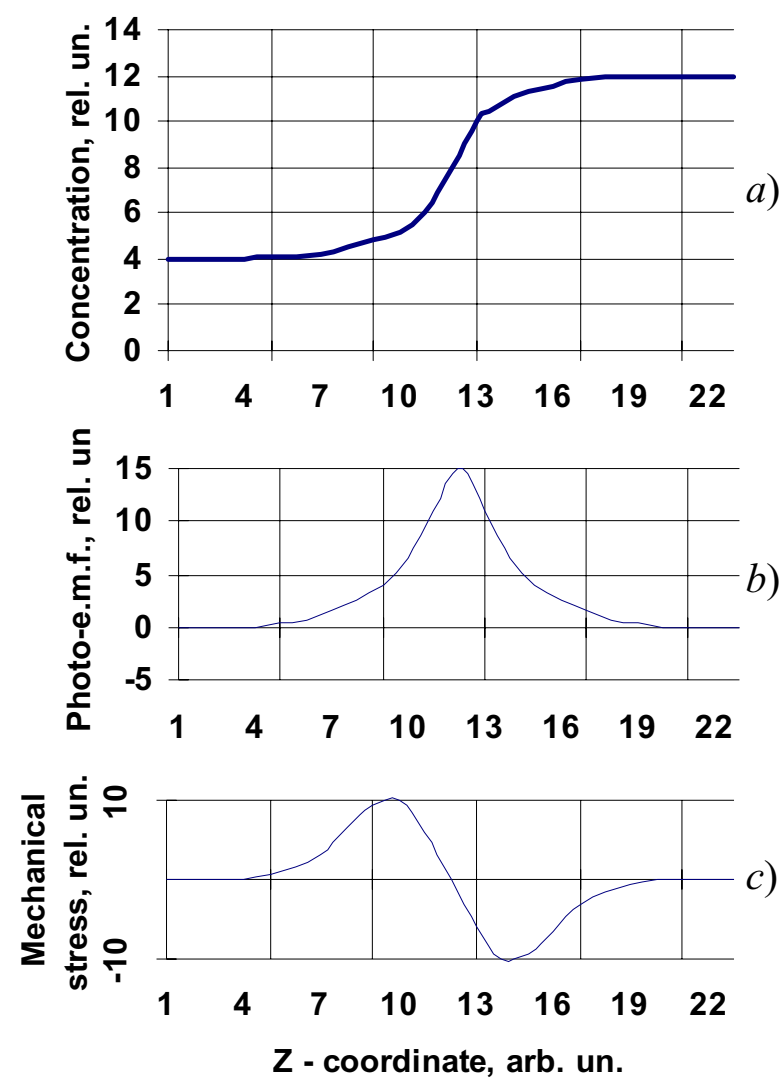

Fig. 1. Distributions of the doping impurity in the crystal (a), of respective volume-gradient photo -e.m.f. $(b)$ and of mechanical stress $(c)$. wide range of electromagnetic waves [1]. Among all these methods the most informative and having the highest detection ability is the method based on measurement of double refraction with modulation of electromagnetic radiation [5]. This is related to the fact that the polarization mode representing the spatial characteristic of the wave is described by a set of parameters, i. e., by components of Maxwell-Jones or Stokes vectors [5]. Therefore, in contrast to modulations of other physical magnitudes dealing with one parameter only, the polarization modulation can be considered as the two-dimensional influence on the wave. It is this fact that provides high information ability of methods and set-up based on the polarization modulation. Besides, the method possessing the advantages of principles of differential spectroscopy, provides higher detective ability in respect to the magnitude of anisotropy of dielectric properties.

It is also worth noting that the macroscopic size of above mentioned nonuniformities makes it possible to apply optopolarization techniques for investigation of their properties. We mean here their spatial resolution ability, when the diameter of the probing beam is less than the characteristic size of nonuniformity, which allows to reliably identify the features of the spatial distribution of nonuniformities.

3 . Since the expected regularity of the voltage dependence on the characteristic of the defect distribution may be of a general character, any semiconductor material can be used for its experimental determination. Here, however, several facts should be taken into account. First of all, the volume-gradient photovoltage should necessarily reflect the actual characteristic of the nonuniformity. To ensure this, the next hierarchy of dimensions should be chosen: $d<L<l$, where $d$ is the diameter of the probing light beam; $L$ is the diffusion length of electron-hole pairs; $l$ is the size of the nonuniformity in the direction of measurements. It is essential that parameters of nonuniformity are stable in the direction normal to the crystal surface, at least, at a distance of the minority carrier diffusion length.

In view of the above mentioned and some other circumstances, a Ge single crystal seems to be the most appropriate material to represent the discussed model. For this purpose the single crystalline ingot was grown using Czochralski method with in-situ formation of $p^{+}-p$-junction by addition into the melt metal In as a dopant. The junction region, $1 \mathrm{~mm}$ thick, was situated between approximately uniform, in respect to their resistivity, parts of the ingot with resistivities of 3 and $0.5 \Omega \times \mathrm{cm}$. The sample was cut from the ingot in the form of a bar with dimensions $I_{\mathbf{z}} \times I_{\mathbf{y}} \times I_{\mathbf{x}}=10 \times 4 \times 1 \mathrm{~mm}$, so that the gradient of nonuniformity was directed along their longest side and the $p^{+}-p$ - junction was situated near the middle of the sample perpendicular to the largest side of the sample. The standard mechanical and chemical treatment was used to fabricate not only a mirror, but also a perfect plane at the sample surface which is necessary for optopolarization measurements. Ohmic contacts at the sample end faces, created by tin welding, were used for con- 


\section{B. K. Serdega et al.: Thermoelasticity in Ge due to nonuniform distribution ...}

nection to the measuring amplifier. The dependence of the volume-gradient photovoltage on the coordinate of the light beam at the sample surface was measured during the sample displacement along the $z$ axis by a conventional technique using the synchronous phase detection.

Regarding the measurement of mechanical stress, in our case the technique was needed which would provide detection of its localization in the near-surface layer with the thickness shorter than the minority carrier diffusion length. As an effective technique of such type one can chose the way used in measurements of double refraction in reflection [5]. This technique is based on the fact (Fig. 2) that, at high values of an absorption index, the reflection of light by a substance occurs in the layer thickness of which is determined by the absorption length. If, in this case, the reflection takes place normally to the surface, then the polarization mode of the light changes caused by anisotropy of dielectric properties induced by the elastic deformation of the crystal (due to an external force or a residual strain) by the magnitude related to the difference of main stress components acting in the plane $z$.
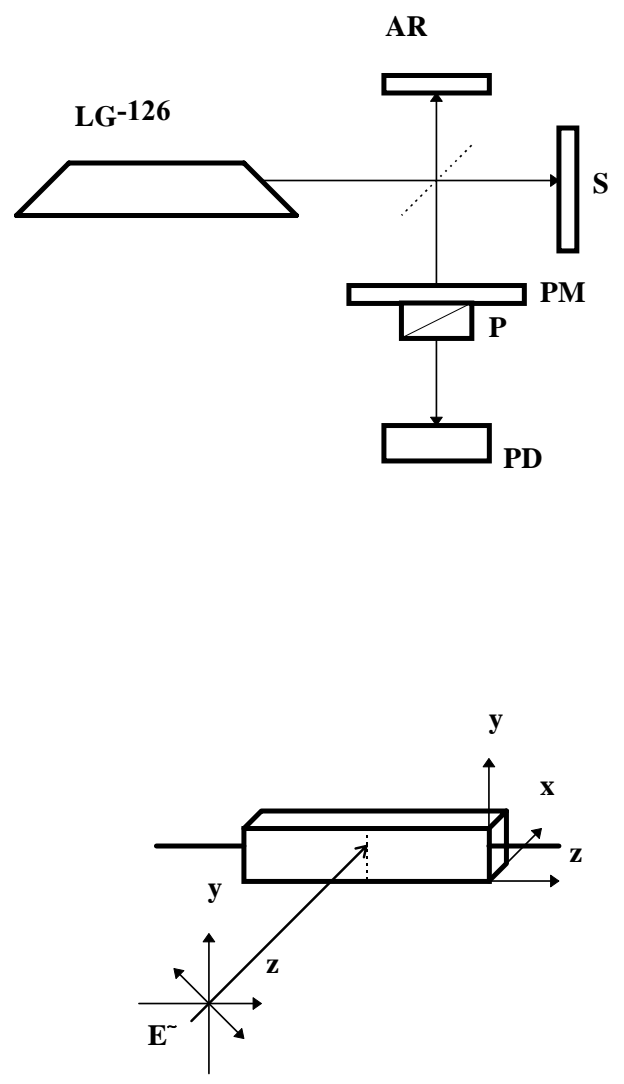

b)

Fig. 2. The optical layout of the experiment $(a)$ and conditions of the sample illumination (b). LG-126 is the He-Ne laser, Sthe sample, $\mathrm{PM}$ is the photoelastic modulator of polarization, $\mathrm{P}$ - the polarizer, $\mathrm{PD}$ - the Ge photodiode, AR is the anisotropic reflector, $E^{\sim}-$ the strength of the electric field of the wave.
The polarization mode of reflected light was analysed by the set-up consisting of the polarization modulator and the linear polarizer, representing, in fact, the dynamic analyser. Since the orientation of optical indicatrix of the sample is known a priori, because it is related to the doping impurity gradient, this fact enabled one to choose the linear polarization mode as an initial mode. Its orientation, in respect to the gradient direction, was set at the angle $45^{\circ}$, and the modulator was tuned, by adjusting the azimuthal position, for messuring the circular component of light. This component, measured by the selective nanovoltmeter at a frequency of modulator, appeared as a part of elliptically polarized, in the general case, reflected light and served as a measure of the berefrigence magnitude, that is, of a deformation value.

As a source of light the He-Ne laser LG-126 was used, the radiation of which at the wavelength $1.15 \mu \mathrm{m}$ is photoactive for Ge crystal, that is, effective for photovoltage generation. The respective absorption coefficient at room temperature, $\alpha$, is of the order of $10^{4} \mathrm{~cm}^{-1}$, and $\alpha^{-1}<L$, which satisfies the condition of the experiment. The latter condition is necessary for reliable detlecting the character of nonuniformity in the photovoltage effect. The laser beam was focused to the light spot with diameter $d \approx 0.1 \mathrm{~mm}$ at the surface of the sample which could be displaced in a controllable manner in respect to the beam along the $z$ axis. Each measurement was completed with the sample returning to the strictly fixed initial position. This allowed one to compare with each other the distributions of the photoelectric and optical signals, which were measured and recorded by plotter.

4. Results of measurements are presented in Fig. 3a, where curves 1 and 2 represent the distributions of the optical signal and of its integral, respectively. In Fig. 3b the curve 2 from Fig. $3 \mathrm{a}$ is shown for comparison with the photovoltage function. It can be seen that, differing in details, the curves in Fig. $3 \mathrm{~b}$ agree in main features, in particular, in positions of maximums. It means that the law $\left|\sigma_{\mathbf{x}}-\sigma_{\mathbf{y}}\right| \sim d^{2} N / d z^{2}$ is actually valid.

Really, the curve 1 in Fig. $3 \mathrm{a}$ is very similar, in its shape, to the plot of voltage in Fig. 1c. At the same time, it can be seen that the actual structure of $p^{+}-p$-junction is, nevertheless, different from the ideal one. So, the photovoltage function is asymmetrical in respect to the junction plane, which can be due to the fact that concentrations, and, in turn, lifetimes at both sides of the junction are strongly different. Besides, the junction itself is more non-monotonic, than it is shown in Fig 1a. This is emphasized by a complicated form of the curve 1 in Fig. 3a, representing the second derivative in respect to concentration, a function which is more sensitive to nonuniformities than the volume-gradient photovoltage represented by the first derivative.

It should be noted that the amplitudes of measured signals and calculated curves are presented in arbitrary units. The matter is that the comparison in absolute units and, therefore, in the same dimensionalities has sense only for results of single integration of the photovoltage function and of double integration of the optical signal. How- 


\section{B. K. Serdega et al.: Thermoelasticity in Ge due to nonuniform distribution ...}

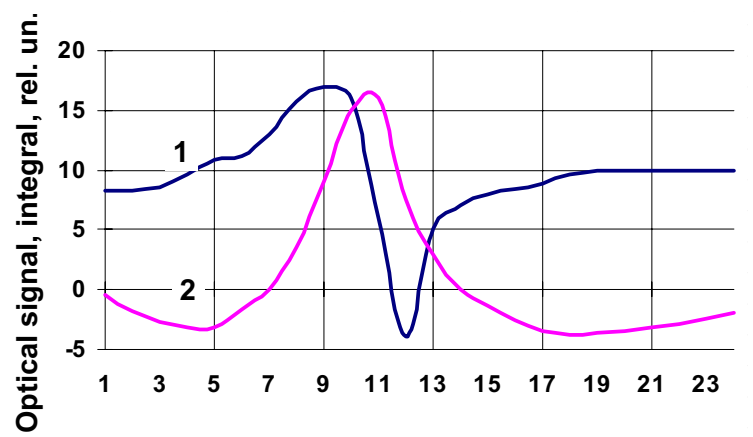

a)

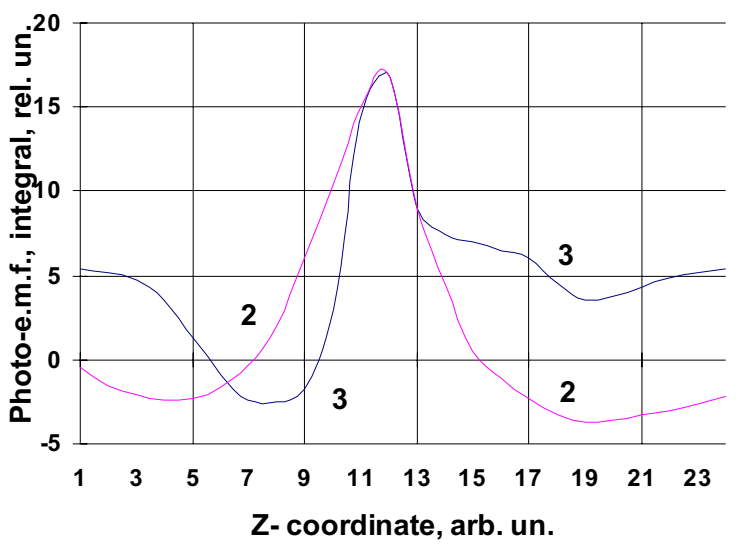

Fig. 3. Distributions, along the doping impurity gradient, of the birefringence signal of double refraction (curve 1), its integral (curve 2) (a) and that of the photo-e.m.f. signal (curve 3) compared to the curve 2 in the Fig. 1a (b).

ever, in this case, the respective constants of integration, one for the photovoltage and two constants for the photoelasticity, must be known. For their determination additional measurements are required, which do not provide new information for the problem solution. The main conclusion drawn from Fig. $3 \mathrm{~b}$ is that the inflection points in the two-dimensional plot of the resistivity form a surface (a plane in the particular case) dividing the regions with different compositions and opposite signs of mechanical stress.

It is of interest to consider the obtained result in respect to the paper [2]. The calculated there coordinate dependencies of mechanical strain on the form of the distribution function of diffusing impurity have the form (in the coordinates of Fig. 2): $\sigma_{x} \sim \exp \left[(a-z)^{2} / 4 D t\right]-$ for Gauss distribution and $\sigma_{\mathbf{x}} \sim \operatorname{erfc}\left[(a-y) /(4 D t)^{1 / 2}\right]$ for the distribution described by the complementary error function (here $a$ is the thickness of the sample, $D, t$ are the coefficient of diffusion and diffusion time, respectively). It should be noted that in both cases the distribution function of stress follows the impurity distribution function. But just this should be expected, if we rely on the regularity ascertained in this work, since the double differen- tiation of exponential functions which were used to characterize the diffusion, does not change their form. And, finally, we should note that the established coordinate dependence of the stress is a consequence of the Poisson equation solution for the displacement $u$ created by the specific force $f$, i.e., by stress [7, p.242]: $\nabla^{2} u=-f / \tau$, where $\tau^{-1}$ is the coefficient of proportionality, the physical sense of which in each specific case is determined by the respective conditions.

Thus, it is found that the nonuniform concentration field, in the same way as the nonuniform temperature field, creates the internal mechanical stress in the solid with a magnitude determined by the function of the spatial distribution of composition. The question about spatial limitations of applicability for this law is still open. This is essential in the case when the macroscopic nonuniformity with decreasing the characteristic size becomes comparable to the interatomic distance, when the field of lattice deformation is created by some discrete defect. We can only suppose that within the magnitudes of deformations which obey the Hooke law, this regularity is valid.

We did not discuss the features related to the impurity (defect) type, namely, to the difference in the coordination radii of the parent atom and of the defect. It is clear that in the above law these features should be manifested in the magnitudes and signs of coefficients of proportionality in the presented relation, i.e., under other equal conditions they should determine the quantitative differences in the stress parameters.

And, finally, we note that the described experiments could be made possible, to the great extent, due to the application of the polarization modulation technique, which allows to register the double refraction in reflection. Therefore, the opticopolarizational method can be applied to the investigations of nontransparent substances, and hence, in a wide range of wavelengths.

\section{References}

1. N. I. Zakharov, A. V. Bagdasarian. Mekhanicheskie yavleniya v integralnykh strukturakh (Mechanical phenomena in the integral structures).- M.: Radio i Sviaz'.- 1992.- p.144 (in Russian).

2. S. Prussin. //J.Appl.Phys. - 32,(18). - p.76 (1961).

3. V. T. Grinchenko, E. V. Nikitenko, B. K. Serdega. Issledovanie metodom poliarizatsionnoy moduliatsii fotouprugogo effecta, indutsirovannogo gradientom temperatury (Investigations by the method of polarization modulation of photoelasticity effect induced by the temperature gradient). Doklady NAN Ukrainy.1998.- No.10.- p.45-49 (in Russian).

4. V. E. Lashkarev, V. A. Romanov. Ob'emnaya fotoeds v poluprovodnikakh (Volume photovoltage in semiconductors) // $R a$ diotekhnika i elektronika.- 1956.- 1, ser. 8.- p.1144-1146 (in Russian).

5. B. K. Serdega. Sposob izmereniya dvoinogo lucheprelomleniya (Technique of measurement of double refraction) Ukrainian Patent No. 19983A of 01.07.94.

6. A. Gerard, C. M. Burch. Vvedenie v matrichnuyu optiku (Introduction to matrix optics).- M: Mir.- 1978.- p.340 (Russian translation).

7. R. Feinman, R. Leiton, M. Sands. Feinman lectures on physics. 5.- 1996.- p.296 (Russian translation). 